\title{
Influences of Powder Source Porosity on Mass Transport during AlN Crystal Growth Using Physical Vapor Transport Method
}

\author{
Danyang Fu ${ }^{1} \oplus$, Qikun Wang ${ }^{2}$, Gang Zhang ${ }^{1}$, Zhe $\mathrm{Li}^{1}{ }^{1}$, Jiali Huang ${ }^{2}$, Jiang Wang ${ }^{1, *}$ and Liang $\mathrm{Wu}^{2, *}$ \\ 1 State Key Laboratory of Advanced Special Steel, Shanghai Key Laboratory of Advanced Ferrometallurgy, \\ School of Materials Science and Engineering, Shanghai University, Shanghai 200072, China; \\ fudanyang@shu.edu.cn (D.F.); zhanggang1996@shu.edu.cn (G.Z.); lizhe1996@shu.edu.cn (Z.L.) \\ 2 Ultratrend Technologies Co., Ltd., Room 518, Building 3, No. 503, Shunfeng Road, Yuhang District, \\ Hangzhou 311199, China; qikun.wang@utrendtech.com (Q.W.); jiali.huang@utrendtech.com (J.H.) \\ * Correspondence: jiangwang@i.shu.edu.cn (J.W.); Jason.wu@utrendtech.com (L.W.)
}

check for updates

Citation: Fu, D.; Wang, Q.; Zhang, G.; Li, Z.; Huang, J.; Wang, J.; Wu, L. Influences of Powder Source Porosity on Mass Transport during AlN Crystal Growth Using Physical Vapor Transport Method. Crystals 2021, 11, 1436. https://doi.org/10.3390/ cryst11111436

Academic Editors: Haiding Sun, M Ajmal Khan, Bharat Jalan,

Shibing Long, Yuhao Zhang,

Rajendra Singh, Xuelin Yang, Yuji Zhao and Bin Liu

Received: 3 November 2021 Accepted: 19 November 2021 Published: 22 November 2021

Publisher's Note: MDPI stays neutral with regard to jurisdictional claims in published maps and institutional affiliations.

Copyright: (c) 2021 by the authors. Licensee MDPI, Basel, Switzerland. This article is an open access article distributed under the terms and conditions of the Creative Commons Attribution (CC BY) license (https:// creativecommons.org/licenses/by/ $4.0 /)$.

\begin{abstract}
We developed a two-dimensional (2D) transport model to investigate mass transport during bulk AlN crystal growth via the physical vapor transport (PVT) process using the finite element method (FEM), taking the powder source porosity, buoyancy, and vapor diffusion into account. The porosity effects of the powder source on mass transport under various growth conditions were investigated in detail. The simulation results show that the porosity of the powder source significantly affects the mass transport process during AlN sublimation growth. When the porosity of the powder source decreases, the growth rate becomes more uniform along the seed deposition surface, although the sublimation rate and crystal growth rate decrease, which can be attributed to the reduced specific surface area of the powder source and the reduced flow rate of Al vapor in the powder source. A flat growth interface can be achieved at a porosity of 0.2 under our specific growth conditions, which in turn facilitate the growth of high-quality AlN crystals and better yield. The decomposition of the powder source and the transport of $\mathrm{Al}$ vapor in the growth chamber can be suppressed by increasing the pressure. In addition, the AlN growth rate variation along the deposition surface can be attributed to the $\mathrm{Al}$ vapor pressure gradient caused by the temperature difference in the growth chamber.
\end{abstract}

Keywords: computer simulation; mass transfer; growth from vapor; AlN single-crystal growth

\section{Introduction}

AlN is a brilliant electronic material for high-temperature, high-frequency, and highpower electronic and optoelectronic devices due to its excellent properties, such as its ultrawide bandgap, high thermal conductivity, high resistivity, strong breakdown voltage, high electron mobility, and low dielectric coefficient [1-3]. AlN has higher lattice and thermal matches and chemical compatibility with $\mathrm{GaN}$ than sapphire or $\mathrm{SiC}$ substrates. Therefore, AlN is an ideal candidate for power devices and sensors, as well as deepUV (DUV) optoelectronic devices with high Al content, such as high-electron-mobility transistors (HEMTs), DUV-LEDs, UV lasers, and solar-blind AlGaN ultraviolet detectors [3]. Physical vapor transport (PVT) has proven to be a very favorable technique for the growth of large-size and high-quality bulk AlN single crystals [4-6]. Nevertheless, the growth conditions required for the preparation of large AlN single crystals are stringent, and the size of the resulting AlN single crystals is still quite limited. At present, the reported maximum size of the obtained AlN single crystal is $60 \mathrm{~mm}$, and only a few research groups have successfully grown $50.8 \mathrm{~mm}$ AlN single crystals [7-9].

The growth of AlN using the PVT method is based on the sublimation of AlN in the source zone and recombination in the crystal growth zone up to $2200-2350{ }^{\circ} \mathrm{C}$. However, performing any extensive experiments in the hostile crystal growth environment is expensive and time-consuming, and numerical simulation has become a powerful tool to better understand the fundamentals behind the growth process. In past decades, many 
research groups have carried out modeling and simulation efforts on AlN crystal growth. Liu et al. [10] developed a global model to study the growth process of AlN considering the surface dynamics and mass transfer. Wu et al. [11-13] evaluated the effects of growth conditions on the crystal growth rate by mass transport modeling and simulation. Gao et al. [14] analyzed the influence of growth parameters on the growth rate using a mass transport model. Wang et al. [15] developed a mass transport model to study the rate limiting step in the crystal growth process, and the results showed the relationship between the Al partial pressure gradient and growth rate under different thermal fields. Fu et al. [16] established mass transfer models considering three-phase $\left(\mathrm{Al} / \mathrm{N} / \mathrm{Al}_{2} \mathrm{O}\right)$ Stefan flow and investigated the influences of growth parameters on oxygen impurity transport in the AlN growth chamber. All above extensive efforts showed that the vapor diffusion and buoyancy have significant effects on mass transport during the AlN crystal growth using the PVT method. To the best of our knowledge, all of the above models assume that the powder source is a nonporous solid, and the mass transport models that consider the effect of powder source porosity on the growth process are rarely reported. Nevertheless, numerous modeling and experimental results during $\mathrm{SiC}$ growth using the PVT method have shown that the powder source porosity has a significant effect on the growth process [17,18]. Therefore, taking into account the powder source porosity in the mass transport model is necessary to accurately predict and better understand the AlN crystal growth using the PVT method.

In this paper, we developed a two-dimensional (2D) transport model to investigate mass transport during bulk AlN crystal growth by the PVT process using the finite element method (FEM). In these models, the effects of the powder source porosity, buoyancy, and vapor diffusion are considered. The temperature distribution is obtained through global modeling technology [19], using the FEMAG two-dimensional axisymmetric model and considering the dual resistance heater. The effects of the powder source porosity on mass transport under various growth conditions are analyzed. The effects of pressure and temperature difference on the crystal growth process considering the porosity of the powder source are investigated in detail.

\section{Geometry Description and Numerical Modeling}

A resistant heating sublimation reactor loaded with an $80 \mathrm{~mm}$ diameter tungsten crucible was used as the AlN crystal growth system, which was mainly composed of two resistant heaters, tungsten heat insulators, and upper and lower infrared temperature measurement systems. For configurations of the furnace, the reader is referred to [15]. During the growth process, the crucible was heated to $2200-2350{ }^{\circ} \mathrm{C}$ in a nitrogen-rich atmosphere. As the powder source decomposed, the mixed gas phase inside the crucible was transported to the seed surface driven by the concentration gradient. For the AlN sublimation crystal growth, the following reaction is considered [10]:

$$
A l(g)+\frac{1}{2} N_{2}(g)=A l N(s) .
$$

In this work, only two species ( $\mathrm{Al}$ and $\mathrm{N}_{2}$ ) in the growth chamber were considered, while impurities such as $\mathrm{Al}_{2} \mathrm{O}$ were neglected due to their low concentrations [10-16]. The powder source charged at the crucible bottom was considered a porous medium. It was assumed that the size of the powder particles was uniform, and the Darcy-BrinkmanForchheimer model was employed in the porous medium [20]. The furnace was assumed to be axisymmetric, and the governing equations in the porous medium were as follows:

$$
\begin{gathered}
\frac{\rho_{f}}{\varepsilon} \frac{\partial \boldsymbol{u}}{\partial t}+\frac{\rho_{f}}{\varepsilon}(\boldsymbol{u} \cdot \nabla) \frac{\boldsymbol{u}}{\varepsilon}=-\nabla p-\rho_{f} \alpha T \boldsymbol{g}+\nabla \cdot\left(\frac{\mu}{\varepsilon} \nabla \boldsymbol{u}\right)-\left(\frac{\mu}{K}+\frac{\rho_{f} b}{K}|\boldsymbol{u}|\right) \boldsymbol{u}, \\
\frac{\partial\left(\varepsilon \rho_{f}\right)}{\partial t}+\nabla \cdot\left(\rho_{f} \boldsymbol{u}\right)=0
\end{gathered}
$$




$$
\frac{\partial\left(\varepsilon C_{i}\right)}{\partial t}+\nabla \cdot\left(C_{i} \boldsymbol{u}\right)-D \varepsilon \nabla^{2} C_{i}+S=0,
$$

where $\varepsilon$ is the porosity of the powder source, $u$ is the velocity field, $\rho_{f}$ is the density of the gas mixture, $\alpha$ is the thermal expansion, $T$ is the temperature of the fluid, $g$ is the gravity vector, $p$ is the system pressure, $\mu$ is the viscosity coefficient, and $C_{i}$ is the molar concentration of species $i$. $D$ is the diffusion coefficient between species $i$, which was obtained from [15]. $S$ denotes the rate of mass transfer of the gas phase from the gas-solid interface to the atmospheric chamber due to the sublimation reaction, which was obtained from [10]. The permeability of porous medium $K$ can be derived as

$$
K=\frac{d_{p}^{2} \varepsilon^{3}}{\left[150(1-\varepsilon)^{2}\right]^{2}}
$$

where $d_{p}$ is the diameter of the particles. The Forchheimer coefficient $b$ can be given by

$$
b=\frac{1.75 K^{0.5}}{\sqrt{150} \varepsilon^{1.5}} .
$$

The governing equations in the growth chamber can be written as

$$
\begin{gathered}
\rho_{f} \frac{\partial \boldsymbol{u}}{\partial t}-\mu \Delta \boldsymbol{u}+\rho_{f}(\boldsymbol{u} \cdot \nabla) \boldsymbol{u}+\nabla p=-\rho_{f} \alpha T \boldsymbol{g} \\
\nabla \cdot \boldsymbol{u}=0 \\
\frac{\partial C_{i}}{\partial t}+\nabla \cdot\left(C_{i} \boldsymbol{u}\right)=D \nabla^{2} C_{i} .
\end{gathered}
$$

The temperature distribution was obtained through global modeling technology [16] using the FEMAG two-dimensional axisymmetric model. Global simulation techniques include an analysis of heat transfer based on decoupled and global-local iterative strategies, taking into account all reactor components (or macroelements) [19]. Two modes of numerical simulations were performed depending on the number of heaters employed, as shown in Figure 1. In Mode I, only the bottom heater was used, and the top center of the powder source $\left(T_{t c}\right)$ was chosen as the temperature control point (TCP). In Mode II, both heaters were used, and the bottom center of the powder source $\left(T_{b c}\right)$ and seed center $\left(T_{s c}\right)$ were chosen as the TCPs. Due to the presence of Knudsen layers at the source and seed surfaces [14-16], the vapor flux at the sublimation and deposition surfaces could be precisely determined by solving the Hertz-Knudsen equation. The deposition or sublimation molar flux is given by

$$
\left(C_{i} \boldsymbol{u}-D \nabla C_{i}\right) \vec{n}=\frac{\gamma_{i}}{\sqrt{2 \pi M_{i} \mathcal{R} T}}\left(p_{i}-p_{i}^{e}\right),
$$

where $\vec{n}$ is the unit normal vector of the surface, which is consistent with the direction of molar flux, and $\gamma_{i}$ and $M_{i}$ are the sticking coefficient and molecular weight of species $i$ taken from [21], respectively. $\mathcal{R}$ is the universal gas constant, and $p_{i}^{e}$ is the equilibrium pressure of species $i$ taken from [22]. The gas velocity at the source-gas interface can be expressed as

$$
u_{-}=u_{+} .
$$

The velocities at the crucible surfaces were all set to zero. For the basic iteration procedures to solve the boundary conditions, details were described in [15] and are not repeated here for brevity. 


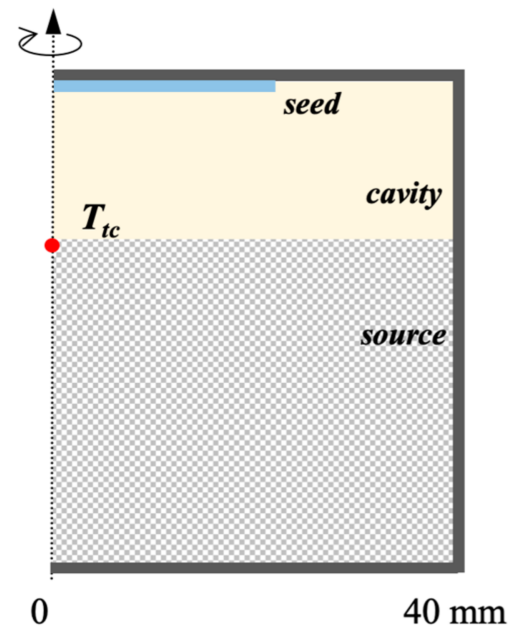

(a)

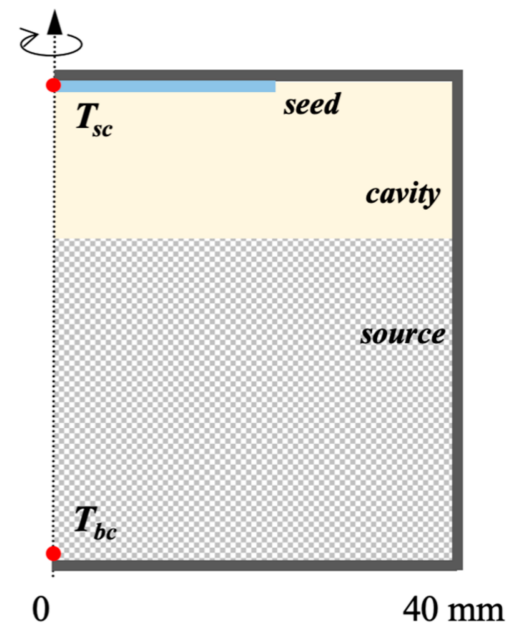

(b)

Figure 1. Schematic of AlN growth chamber. (a) Mode I: the top center of the powder source as TCP. (b) Mode II: the seed center and bottom center of the powder source as two TCPs.

\section{Validation of the Model}

Two sets (cases 1 and 2) of practical growth experimental data from our team were used to validate the developed models, as shown in Figure 2. We can see that the simulation results were fairly consistent with these experimental results. The growth conditions were as follows: (1) Mode I (ref Figure 1a) was used for thermal field control, with the top center of the powder source at $2200{ }^{\circ} \mathrm{C}$, pressure of $50 \mathrm{kPa}$, and source-seed distance of $20 \mathrm{~mm}$; (2) Mode II (ref Figure $1 \mathrm{~b}$ ) was used, with one TCP $\left(T_{s c}\right)$ set as $2200^{\circ} \mathrm{C}$ and the other TCP $\left(T_{b c}\right)$ set as $2300^{\circ} \mathrm{C}$, pressure of $70 \mathrm{kPa}$, and source-seed distance of $20 \mathrm{~mm}$.

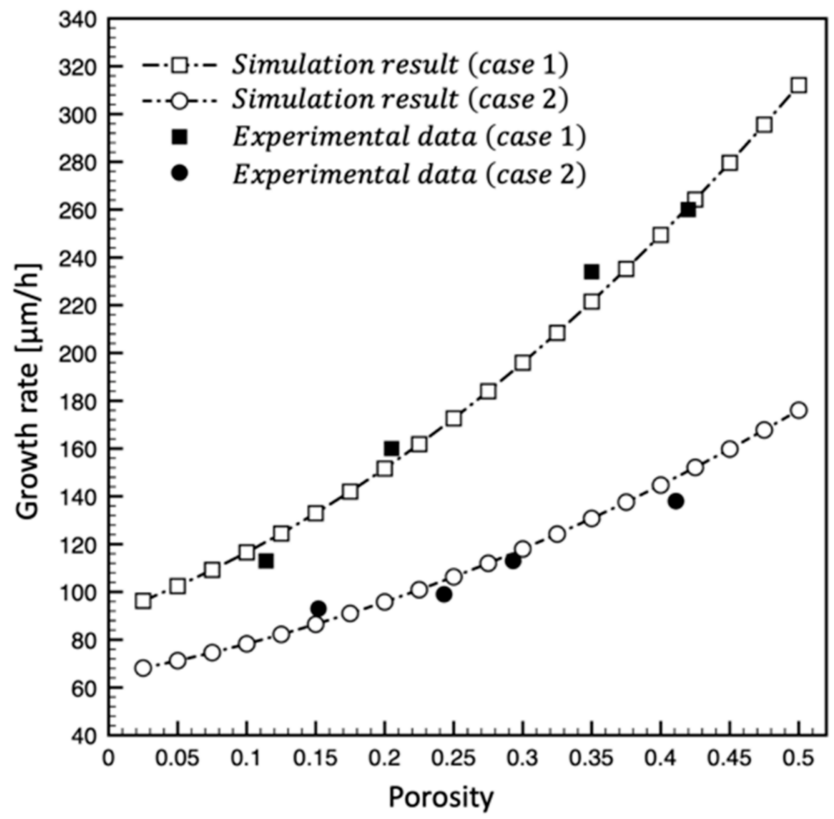

Figure 2. Growth rate comparison between the numerical and experimental data for both cases.

\section{Simulation Results and Discussion}

\subsection{Effect of the Powder Porosity}

It is well known that mass transport in the powder source has a great influence on the sublimation growth process. A series of simulations were carried out to investigate the effect of the powder porosity on the sublimation rate and crystal growth rate. In these 
simulations, Mode I was used for thermal field control, with the top center of the powder source at $2200{ }^{\circ} \mathrm{C}$. The atmospheric pressure was set to $50 \mathrm{kPa}$. The height of the powder source and the source-seed distance were set to $60 \mathrm{~mm}$ and $20 \mathrm{~mm}$, respectively.

The sublimation rates at the top surface of the powder source and the growth rates at the seed surface under various powder porosities are presented in Figure 3. The simulation results clearly show that an increase in the initial porosity of the powder source could significantly increase the sublimation rate and the growth rate. Figure 4 shows the distribution of the mixed flow field and $\mathrm{Al}$ vapor partial pressure in the growth chamber at powder source porosities of $0.4,0.3,0.2$, and 0.1 . The $\mathrm{Al}$ gas was transferred inside the powder source and then to the top surface. With increasing porosity, the flow rate increased significantly. When the porosity increased, the sublimation rate increased significantly for two reasons. One reason is that the specific surface area of the powder source increased significantly due to the increase in porosity. Another reason is that a larger porosity was beneficial to the transport of $\mathrm{Al}$ vapor inside the powder source to the evaporation surface of the powder. In addition, attention should be given to the effect of the powder source porosity on the growth uniformity, since the growth of large-size AlN crystals requires a uniform growth rate along the seed surface to achieve high structural quality [14] and better yield. When the porosity decreased, the growth rate became more uniform along the seed surface, although the sublimation rate and growth rate decreased. When the porosity was 0.2 under our specific PVT system and growth conditions, the difference between the maximum growth rate at the edge of the deposition interface and the central growth rate $\left(\Delta V_{g}\right)$ was less than $10 \mu \mathrm{m} / \mathrm{h}$, and the growth rate reached more than $200 \mu \mathrm{m} / \mathrm{h}$. These results imply that a flat growth interface could be obtained while maintaining a high growth rate.

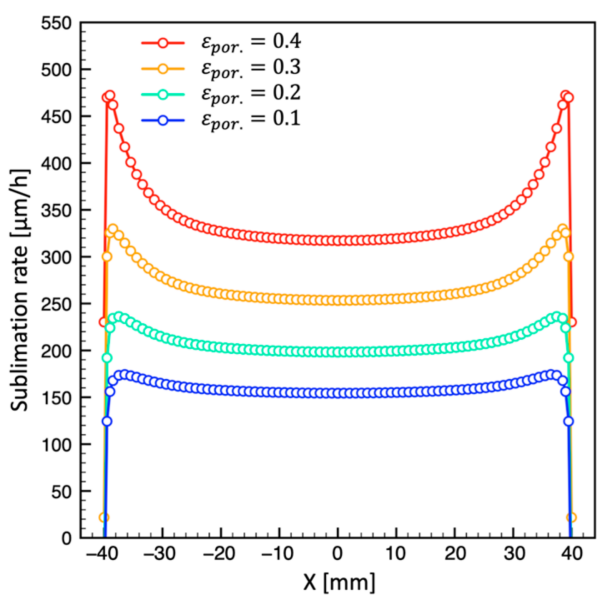

(a)

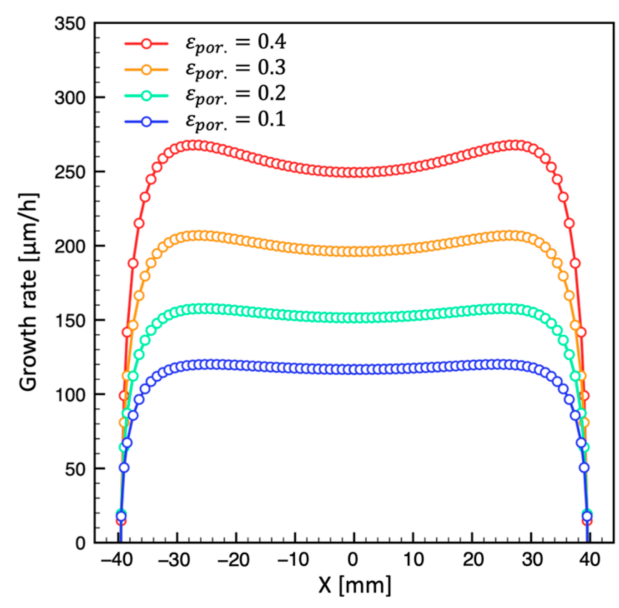

(b)

Figure 3. Profiles of the sublimation rate along the top surface of the powder source (a) and growth rate along the seed surface $(\mathbf{b})$ at various porosities.

\subsection{Effect of Pressure}

As pointed out in many studies $[10,23,24]$, the total pressure in the growth chamber has a significant effect on AlN crystal growth. To further clarify the influences of pressure on mass transport and growth rate, a series of simulations were carried out using different pressures while fixing other growth conditions. In these simulations, Mode I was used, and the top center of the powder source was set to $2200{ }^{\circ} \mathrm{C}$. The porosity of the powder source was set to 0.4 , and the height of the powder source and the source-seed distance were set to $60 \mathrm{~mm}$ and $20 \mathrm{~mm}$, respectively. 


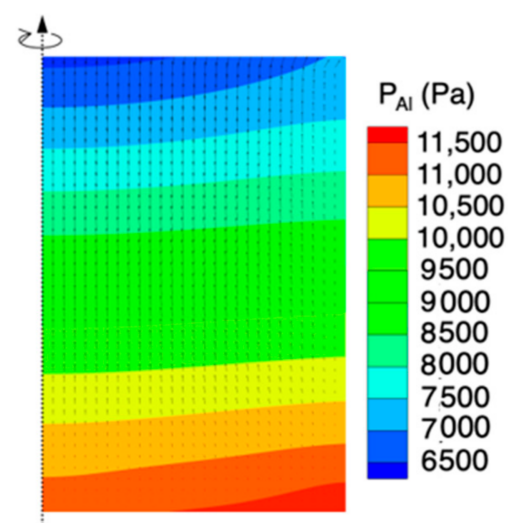

(a)

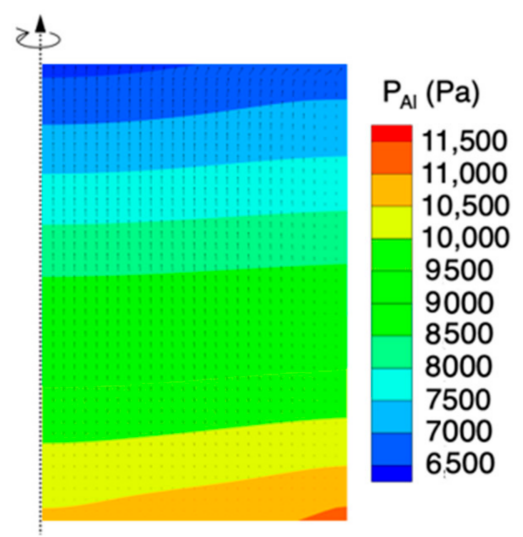

(c)

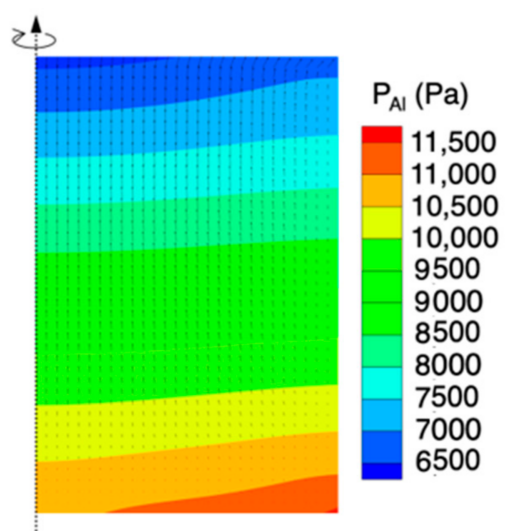

(b)

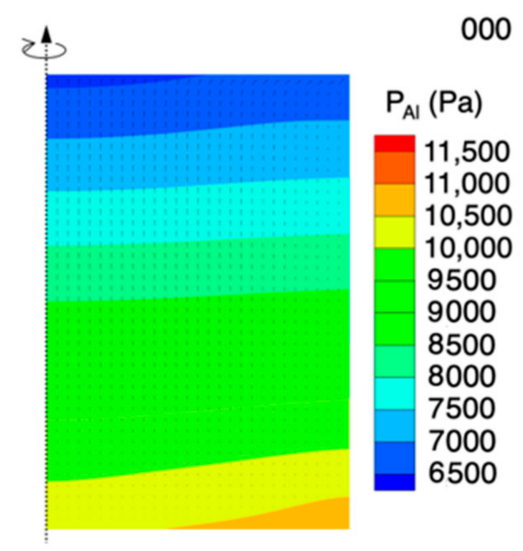

(d)

Figure 4. Distribution of mixed flow and Al vapor partial pressure in the growth chamber at porosities of (a) 0.4 , (b) 0.3 , (c) 0.2 , and (d) 0.1 .

The profiles of the growth rate and sublimation rate at various pressures predicted by the developed models are illustrated in Figure 5. The sublimation rate and growth rate were reduced nearly threefold as the pressure increased from $50 \mathrm{kPa}$ to $90 \mathrm{kPa}$. This can be attributed to the suppression of Al vapor sublimation and transmission at high atmospheric $\left(\mathrm{N}_{2}\right)$ pressure, as shown in Figure 6. To further study the influence of pressure on the uniformity of the growth rate, the $\Delta V_{g}$ and the effective diameter $D_{e f f}\left(\Delta V_{g} \leq 1 \mu \mathrm{m} / \mathrm{h}\right)$ under various pressures were compared, as shown in Figure 7. As the pressure increased, $\Delta V_{g}$ gradually decreased, and $D_{\text {eff }}$ progressively increased. High pressure was suitable for obtaining a uniform initial growth interface. When the pressure inside the furnace reached $90 \mathrm{kPa}$, the effective diameter reached a maximum of $20 \mathrm{~mm}$.

\subsection{Effect of Temperature Difference}

The temperature difference $\Delta T$ between the sublimation surface and seed deposition surface plays an important role in the AlN growth rate [14]. To analyze the effect of temperature difference $\Delta T$ on the AlN sublimation growth process, numerical experiments were carried out by precisely regulating the $\Delta T$ between the seed center $T_{S C}$ and the bottom center of the powder source $T_{b c}$, i.e., $\Delta T=T_{s c}-T_{b c}$. In these simulations, Mode II (see Figure $1 b$ ) was used, where one TCP $T_{s c}$ was fixed at $2200{ }^{\circ} \mathrm{C}$, and the other TCP $T_{b c}$ was changed to control the temperature gradient inside the crucible at $1-1.6^{\circ} \mathrm{C} / \mathrm{mm}$. The porosity of the powder source was set to 0.3 , and the atmospheric pressure was set to $70 \mathrm{kPa}$. 


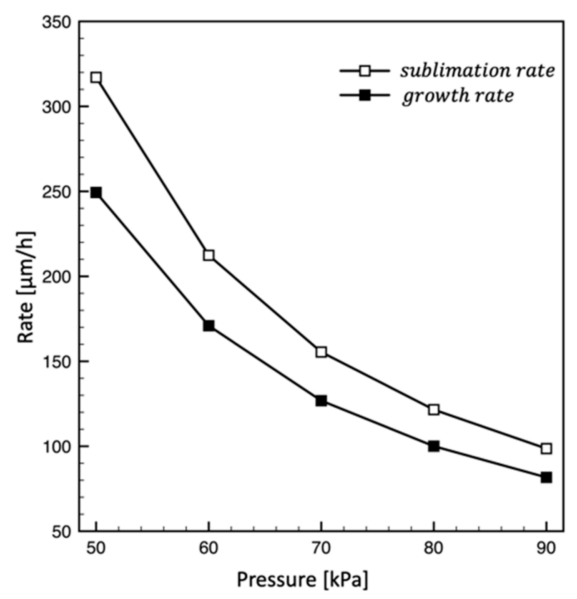

Figure 5. Profiles of the sublimation rate and growth rate under various pressures.

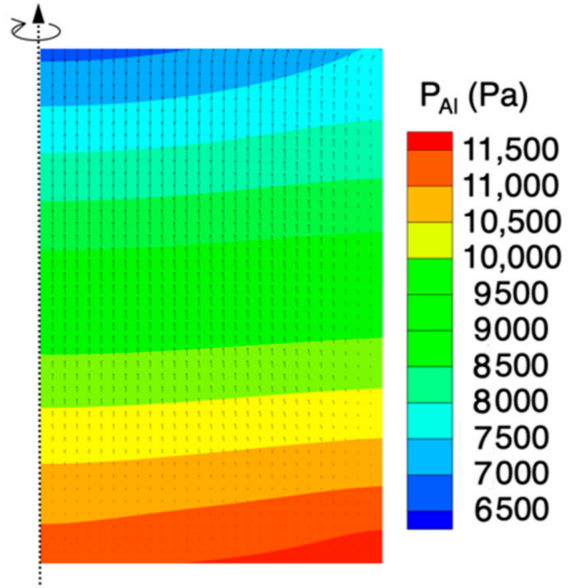

(a)

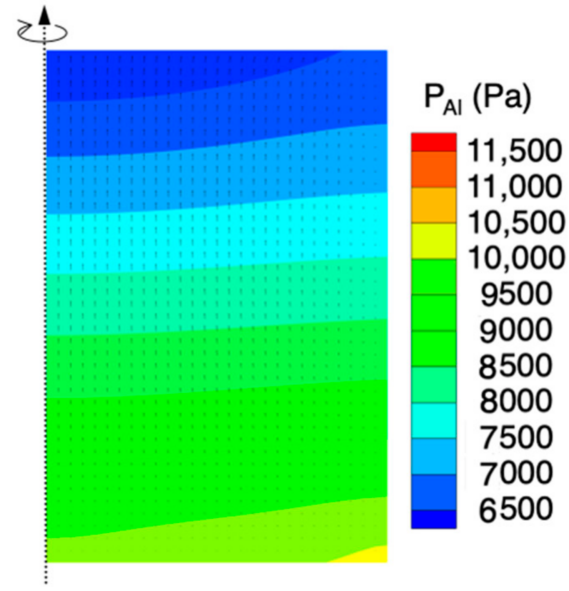

(b)

Figure 6. Distribution of mixed flow and $\mathrm{Al}$ vapor partial pressure in the growth chamber for pressures of (a) $50 \mathrm{kPa}$ and (b) $90 \mathrm{kPa}$.

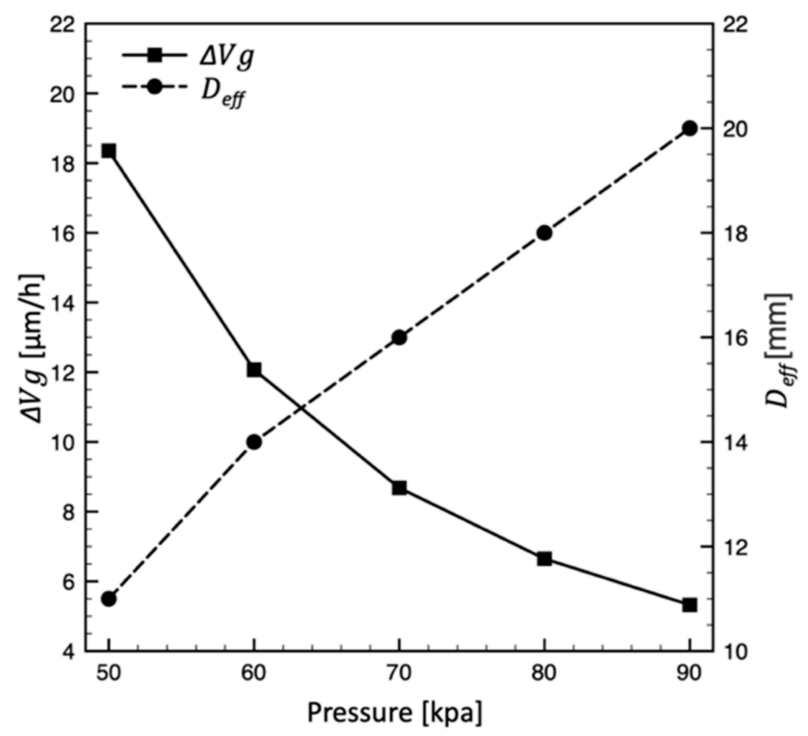

Figure 7. Differences in the maximum growth rate and the minimum growth rate $\left(\Delta V_{g}\right)$ and effective diameter $D_{\text {eff }}$ with different pressures. 
The profiles of the sublimation rate on the top surface of the powder source and the growth rate on the seed surface under various temperature differences $\Delta T$ are shown in Figure 8 . The sublimation rate and growth rate decreased rapidly with decreasing $\Delta T$, and the growth interface tended to be flat at lower $\Delta T$. Figure 9 shows the mixed flow field and the $\mathrm{Al}$ vapor partial pressure distribution in the growth chamber at different $\Delta T$. $\mathrm{A}$ large $\Delta T$ was beneficial to the decomposition of the powder source and, therefore, could promote mass transfer inside the growth chamber and increase the crystal growth rate. It is well known that the equilibrium pressure difference between the powder source and the growing crystal is a key parameter of the growth rate [14]. Wang et al. [15] revealed that the $\mathrm{Al}$ vapor pressure gradient is the limiting step of AlN growth. The profiles of the growth rate and $\mathrm{Al}$ vapor partial pressure gradient along the seed surface at $\Delta T=80^{\circ} \mathrm{C}$, $100{ }^{\circ} \mathrm{C}$, and $120^{\circ} \mathrm{C}$ are illustrated in Figure 10. It can be observed that the $\mathrm{Al}$ vapor pressure gradient increased with increasing temperature difference, as in the case of the growth rate. The $\mathrm{Al}$ vapor pressure gradient on the seed surface showed a very similar trend to the growth rate; therefore, the variation in the growth rate could be attributed to the $\mathrm{Al}$ vapor pressure gradient caused by the temperature difference in the growth chamber.

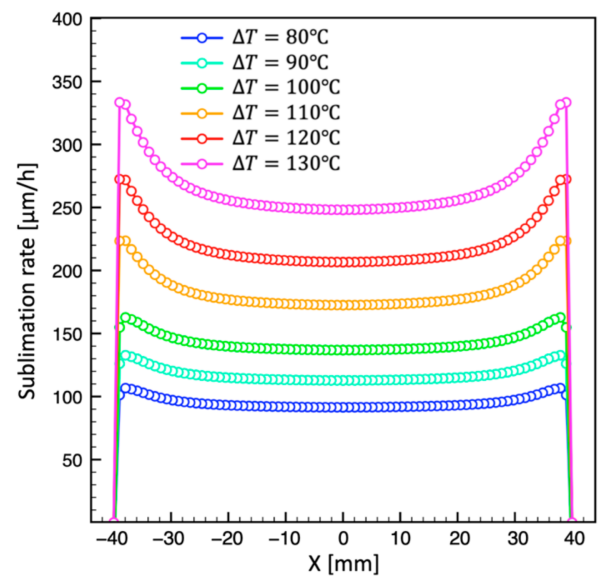

(a)

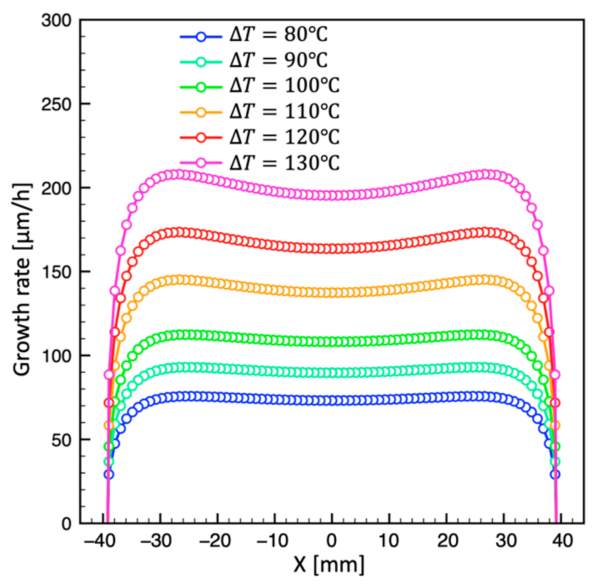

(b)

Figure 8. Profiles of the sublimation rate along the top surface of the powder source (a) and the growth rate along the seed surface (b) at different $\Delta T$.

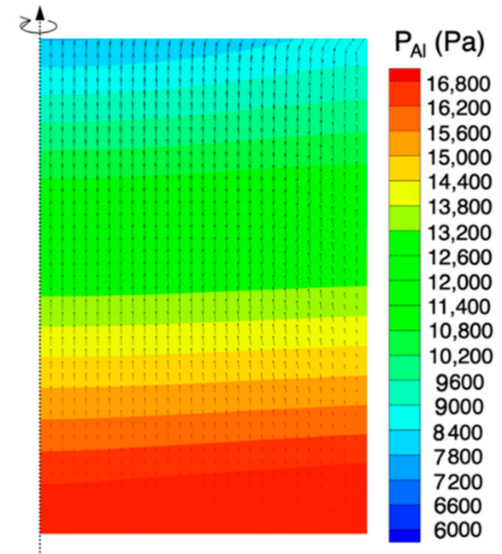

(a)

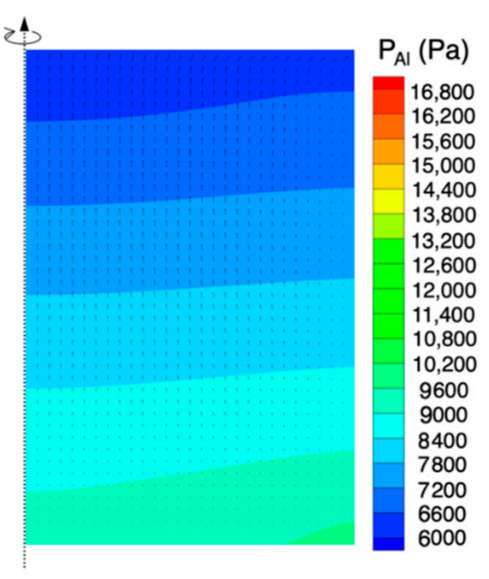

(b)

Figure 9. Mixed flow field together with $\mathrm{Al}$ vapor partial pressure in growth chamber at $\Delta T=130{ }^{\circ} \mathrm{C}(\mathbf{a})$ and $\Delta T=80^{\circ} \mathrm{C}(\mathbf{b})$. 


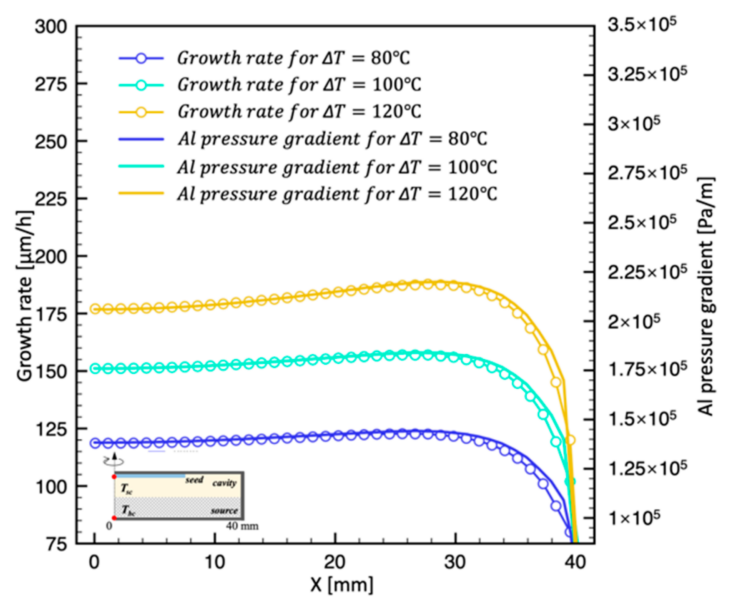

Figure 10. Profiles of growth rate and $\mathrm{Al}$ vapor partial pressure gradient along the seed surface at $\Delta T=80^{\circ} \mathrm{C}, 100^{\circ} \mathrm{C}$, and $120^{\circ} \mathrm{C}$.

\section{Conclusions}

A 2D incompressible flow and mass transport model was developed to investigate the process of mass transfer during bulk AlN crystal growth by the PVT growth process using the FEM method, taking the effects of powder source porosity, buoyancy, and vapor diffusion into account. The influences of the powder source porosity on the mass transport under various growth conditions were investigated in detail. The simulation results showed that the powder source porosity significantly affected the mass transfer of the AlN sublimation growth. When the porosity of the powder source decreased from 0.4 to 0.1 under our specific PVT system and growth conditions, the growth rate became more uniform along the seed deposition surface, although the crystal growth rate decreased from $250 \mu \mathrm{m} / \mathrm{h}$ to $110 \mu \mathrm{m} / \mathrm{h}$, which could be attributed to the reduced specific surface area of the powder source and the reduced flow rate of $\mathrm{Al}$ vapor in the powder source. When the porosity was 0.2 , a flat growth interface $\left(\Delta V_{g}<10 \mu \mathrm{m} / \mathrm{h}\right)$ could be obtained while maintaining a high growth rate $(>200 \mu \mathrm{m} / \mathrm{h})$. The simulation results also show that increasing the pressure suppressed the sublimation and transport of $\mathrm{Al}$ vapor, which allowed the growth rate and effective diameter to be in the range of $70-250 \mu \mathrm{m} / \mathrm{h}$ and $5.5-20 \mathrm{~mm}$, respectively. When the pressure inside the furnace reached $90 \mathrm{kPa}$, the effective diameter reached a maximum of $20 \mathrm{~mm}$. In addition, the simulation results revealed that the AlN growth rate variation along the deposition surface could be attributed to the $\mathrm{Al}$ vapor pressure gradient caused by the temperature difference in the growth chamber.

Author Contributions: Conceptualization, J.W. and L.W.; methodology, D.F. and G.Z.; software, D.F. and Q.W.; writing — original draft preparation, D.F. and Q.W.; writing—review and editing, Z.L. and J.H.; supervision, L.W. All authors have read and agreed to the published version of the manuscript.

Funding: This work was supported by the National Natural Science Foundation of China (61874071) and the Key Research and Development Program of Zhejiang Province (2020C01145).

Institutional Review Board Statement: Not applicable.

Informed Consent Statement: Not applicable.

Data Availability Statement: The data presented in this study are available within the article.

Acknowledgments: The authors thank Dan Lei and Yinting Zhao for providing the experimental data.

Conflicts of Interest: The authors declare no conflict of interest. 


\section{References}

1. Ambacher, O. Growth and applications of Group III-nitrides. J. Phys. D Appl. Phys. 1998, 31, 2653-2710. [CrossRef]

2. Fu, D.; Gong, J.; Lei, D.; Huang, J.; Wang, Q.; Wu, L. Recent Progress and Future Challenges of AlN Single Crystal Growth by Physical Vapor Transport. J. Synth. Cryst. (Chin.) 2020, 49, 1141-1156.

3. Hartmann, C.; Dittmar, A.; Wollweber, J.; Bickermann, M. Bulk AlN growth by physical vapour transport. Semicond. Sci. Technol. 2014, 29, 084002. [CrossRef]

4. Noveski, V.; Schlesser, R.; Raghothamachar, B.; Dudley, M.; Mahajan, S.; Beaudoin, S.; Sitar, Z. Seeded growth of bulk AlN crystals and grain evolution in polycrystalline AlN boules. J. Cryst. Growth 2005, 279, 13-19. [CrossRef]

5. Epelbaum, B.M.; Bickermann, M.; Nagata, S.; Heimann, P.; Filip, O.; Winnacker, A. Similarities and differences in sublimation growth of SiC and AlN. J. Cryst. Growth 2007, 305, 317-325. [CrossRef]

6. Sumathi, R.R. Native seeding and silicon doping in bulk growth of AlN single crystals by PVT method. Phys. Status Solidi C 2014, 11, 545-548. [CrossRef]

7. Wang, Q.; Lei, D.; He, G.; Gong, J.; Huang, J.; Wu, J. Characterization of 60 mm AlN Single Crystal Wafers Grown by the Physical Vapor Transport Method. Phys. Status Solidi A 2019, 1900118. [CrossRef]

8. Chemekova, T.Y.; Avdeev, O.V.; Barash, I.S.; Mokhov, E.N.; Nagalyuk, S.S.; Roenkov, A.D.; Segal, A.S.; Makarov, Y.N.; Ramm, M.G.; Davis, S.; et al. Sublimation growthof 2 inch diameter bulk AlN crystals. Phys. Status Solidi C 2008, 5, 1612-1614. [CrossRef]

9. Schujman, S.B.; Schowalter, L.J.; Bondokov, R.T.; Morgan, K.E.; Liu, W.; Smart, J.A.; Bettles, T. Structural and surface characterization of large diameter, crystalline AlN substrates for device fabrication. J. Cryst. Growth 2008, 310, 887-890. [CrossRef]

10. Liu, L.; Edgar, J.H. A Global Growth Rate Model for Aluminum Nitride Sublimation. J. Electrochem. Soc. 2002, 149, G12-G15. [CrossRef]

11. Wu, B.; Ma, R.; Zhang, H.; Dudley, M.; Schlesser, R.; Sitar, R. Growth kinetics and thermal stress in AlN bulk crystal growth. J. Cryst. Growth 2003, 253, 326-339. [CrossRef]

12. Wu, B.; Zhang, H. Transport phenomena in an aluminum nitride induction heating sublimation growth system. Int. J. Heat Mass Transfer 2004, 47, 2989-3001. [CrossRef]

13. Wu, B.; Ma, R.; Zhang, H.; Prasad, V. Modeling and simulation of AlN bulk sublimation growth systems. J. Cryst. Growth 2004, 266, 303-312. [CrossRef]

14. Gao, B.; Nakano, S.; Kakimoto, K. The impact of pressure and temperature on growth rate and layer uniformity in the sublimation growth of AlN crystals. J. Cryst. Growth 2012, 338, 69-74. [CrossRef]

15. Wang, Q.; Huang, J.; Fu, D.; He, G.; Lei, D.; Wu, L. Influence of crucible shape on mass transport in AlN crystal growth by physical vapor transport process. J. Cryst. Growth 2019, 515, 21-25. [CrossRef]

16. Fu, D.; Wang, Q.; Zhang, G.; Zhu, R.; Liu, H.; Li, Z.; Wu, L. Modelling and simulation of oxygen transport during AlN crystal growth by the PVT method. J. Cryst. Growth 2020, 551, 12592. [CrossRef]

17. Wellmanna, P.J.; Hofmanna, D.; Kadinskib, L.; Selderb, M.; Straubingera, T.L.; Winnacker, A. Impact of source material on silicon carbide vapor transport growth process. J. Cryst. Growth 2001, 225, 312-316. [CrossRef]

18. Wang, X.; Cai, D.; Zhang, H. Increase of SiC sublimation growth rate by optimizing of powder packaging. J. Cryst. Growth 2007, 305, 122-132. [CrossRef]

19. Dupret, F.; Nicodème, P.; Ryckmans, Y.; Wouters, P.; Crochet, M.J. Global modelling of heat transfer in crystal growth furnaces. Int. J. Heat Mass Transfer 1990, 33, 1849-1871. [CrossRef]

20. Khoei, A.R.; Salehi, S.A.; Hosseini, N. Modeling of reactive acid transport in fractured porous media with the Extended-FEM based on Darcy-Brinkman-Forchheimer framework. Comput. Geotech. 2020, 128, 103778. [CrossRef]

21. Mueller, S.G.; Bondokov, R.T.; Morgan, K.E.; Slack, G.A.; Schujman, S.B.; Grandusky, J.; Smart, J.; Schowalter, L.J. The progress of AlN bulk growth and epitaxy for electronic applications. Phys. Status Solidi A 2009, 206, 1153-1159. [CrossRef]

22. Liu, L.; Edgar, J.H. Transport effects in the sublimation growth ofaluminum nitride. J. Cryst. Growth 2000, 220, 243-253. [CrossRef]

23. Karpov, S.Y.; Zimina, D.V.; Makarov, Y.N.; Mokhov, E.N.; Roenkov, A.D.; Ramm, M.G.; Vodakov, Y.A. Sublimation Growth of AlN in Vacuum and in a Gas Atmosphere. Phys. Status Solidi A 1999, 176, 435-438. [CrossRef]

24. Wolfson, A.A.; Mokhov, E.N. Dependence of the Growth Rate of an AlN Layer on Nitrogen Pressure in a Reactor for Sublimation Growth of AlN Crystals. Semiconductors 2010, 44, 1430-1432. [CrossRef] 